\title{
Improving the Transmission Capacity Using Dwt as Multicarrier Modulation for STBC MIMO System
}

\author{
${ }^{1}$ S.Kalyanarajan and ${ }^{2}$ S.Krithiga, Assistant professor \\ ${ }^{1,2}$ Department of ECE, SRM University, Kattankulathur -603203, Tamil Nadu, India
}

\begin{abstract}
The transmission through wireless channel suffers from many challenges due to the multipath effect that causes Inter Symbol Interference (ISI) problem. Multicarrier modulation (MCM) is proposed as solution to overcome the ISI. This paper presents the Discrete Wavelet Transform (DWT) based multicarrier modulation as alternative platform of conventional OFDM in which there is no need for cyclic prefix overhead due to the overlapping nature of DWT. Simulation based analysis will be used to simulate the two multicarrier systems, DWT with 'haar' mother based multicarrier STBC-MIMO system (The term multicarrier refers OFDM), in which information is conveyed with an STBC matrix that is transmitted from combinations of the transmit antennas of the corresponding MIMO system, and the conventional STBC-MIMO under the scenario of having multiple antennas system, with MQAM and MPSK as two modulation schemes in Rayleigh fading channel. Based on the bit error rate performance and the transmission capacity, the DWT based multicarrier system was found to be superior compared with the conventional OFDM system.
\end{abstract}

Index Terms: OFDM (Orthogonal Frequency Division Multiplexing), STBC (Space Time Block Code), MIMO (Multi Input Multi Output), M-QAM (M-ary quadrature amplitude modulation), M-PSK (M-ary phase shift keying)

\section{Introduction}

The use of multiple antennas at the transmitter and receiver in wireless systems, popularly known as MIMO (multiple-input multiple-output) technology, has rapidly gained in popularity over the past decade due to its powerful performance-enhancing capabilities. The technology offers a number of benefits that help meet the challenges posed by both the impairments in the wireless channel as well as resource constraints. [1]The DWTOFDM has to satisfy the orthonormal basis and the perfect reconstruction properties to be considered for OFDM. Different wavelet families have been used and BER performance is compared with the conventional FFT -OFDM system for AWGN. There are several advantages of using wavelets for wireless communication systems [2] the Fourier transform is only suitable for signals whose frequency content does not change with time. The Fourier transform does not tell at which time these frequency component occurs. To solve this problem, the wavelet transform, which was developed to provide a better time frequency representation of the signal than any other existing transform [3] .multi-carrier (MC) orthogonal CDM A signaling scheme was introduced as a mean to reduce interference in multi-point to point CDMA network. It presents a class of spreading codes that enables a simple de-spreading combining receiver to achieve the performance of optimum multi-user linear receiver. The main advantages of the Multicarrier Modulation are; firstly to solve the multipath propagation problem using simple equalization at the receiver. Secondly, the system offers computation efficiency [4] an efficient method to obtain high- rate ,full- diversity STBCs with simplified ML decoding , and applied to STBC designs for CIODs and it is possible to obtain high-rate STBCs with significantly lower decoding complexities without degradation in error performance [5] conditions for reducing complexity ML decoding and applied them to united analysis or two families of full diversity $2 \times 2$ STBCs that were recently proposed [6] a full rate code selection technique has been proposed to improve the error performance of the wireless communication systems within transmit and one receive antennas where $\mathrm{n}>2$.

The approach aims to maximize the diversity by choosing the appropriate STBC for each possible channel situation from a set of balanced STBCs at the transmitter [7] the performance of SM degrades in the presence of a LOS component, whereas the performance of Alamouti improves in such channel condition. Another limitation of SM is the increase in spectral efficiency by the base-two logarithm of the total number of transmits antennas, as compared to a line are increase for the V-BLAST system [8] Spatial modulation avoids ICI at the receiver input, produces no correlation between the transmit antennas and requires no synchronization between them [9] the rate of an orthogonal design is defined as the ratio of the number of transmitted information symbols in a block of channel uses to the length of the given block, which reflects the bandwidth efficiency of the employed space-time block code constructed from the orthogonal design [10]the performance gain obtained by increasing the number of transmit antennas in STBC systems, especially in channels with a large Doppler spread. In multi- path channels, the training overhead associated with increasing the number of transmit antennas was shown to be so large that no benefit is obtained by using STBC [11] high Peak to 
Average Power Ratio(PAPR) of the transmitted signal is a major problem in Orthogonal Frequency Division Multiplexing(OFDM)which induces the degradation of bit error rate(BER) leading to a significant loss in the transmission power efficiency [12] it is found that the BER performance of the system decreases on increase in modulation order. Performance of STBC- OFDM get improves with unequal power conditions and with antenna selection technique. The system performs well in slow fading (even very slow fading). If fading is somewhat rapid, it does not achieve good performance.[13] Space-Time Coding was provided by presenting Alamouti's scheme and discussed block codesschemeswithdifferentcoderatesforthecasesof3 and4transmitantennas.

\section{System Description}

Wavelet transform is a mathematical function that is emerged as a new tool for multi-resolution decomposition of continuous time signal at different times and different frequencies (or scales). So the wavelet transform provides the time-frequency representation of the signal. In wavelet transform, higher frequencies are better resolved in time, and lower frequencies are better resolved in frequency. In this sense, the signal is multiplied with an orthogonal wavelet function, and the transform can able to compute separately for different segments of the time domain signal. The continuous wavelet transform can be defined as

$$
C W T_{\chi}^{\psi}(\tau, \mathrm{s})=\frac{1}{\sqrt{|s|}} \int_{-\infty}^{\infty} x(t) \psi\left(\frac{t-\tau}{s}\right) d t \quad \rightarrow(1)
$$

Where:CWT $T_{\chi}^{\psi}$ is the Continuous wavelet transform of the signal $x(t), \psi(t)$ is the mother wavelet, $\tau$ represents the translation parameter which is corresponding to the time information in the signal and ' $\mathrm{S}$ ' represents the Scale parameter which is corresponding to the frequency information in the signal, Scale = $1 /$ frequency.

The modified version of the wavelet transform is the discrete wavelet transform (DWT), in which the scaling as well as the translation variables is properly discretized. If the scaling variable ' $\mathrm{S}$ ' is discretized by $2^{\mathrm{m}}$ and the translation variable" $t$ " by $2^{\mathrm{m}} \mathrm{n}$, where and are integers, then the DWT of a continuous-time signal $\mathrm{s}(\mathrm{t})$ is expressed as

$$
s_{n}^{m}=2^{-m / 2} \int s(t) \psi\left(2^{-m} t-n\right) d t
$$

Where: $s_{n}^{m}$ represent the DWT coefficients at different scale and translation variables

\section{a. FFT-OFDM System design}

Multicarrier modulation (MCM) is an effective technique of wireless communication; unlike traditional single carrier systems it divides the bandwidth into independent narrow sub-band channels, and uses it to transmit parallel low data streams which are divided from the original high data stream. In Fig.1 the number of sub-channels is chosen to make the symbol time for each sub-stream much greater than the delay spread of the channel and to make the sub-stream bandwidth less than the channel coherence bandwidth to avoid the ISI. Multicarrier modulation provides an efficient means to access and distribute multiple multiplexed data streams. This is potentially very attractive for high speed, broadband networks, which often multiplex multiple data sources during transmission. The traditional multicarrier modulation scheme is the Orthogonal Frequency Division Multiplexing (OFDM) in which the Discrete Fourier Transform (DFT) is used to construct the orthogonal subcarriers.

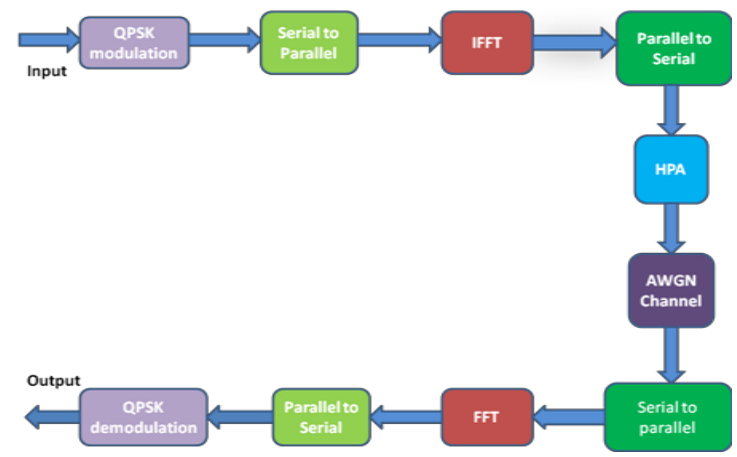

Fig.1.FFT-BasedOFDMSystem 
OFDM is emerged to combat the inter symbol interference (ISI), channel distortion and improve the spectral efficiency, ISI is eliminated in OFDM by added a cyclic prefix to the OFDM signal but this can decrease the bandwidth efficiency greatly. In conventional OFDM transceiver the Inverse Fast Fourier Transform (IFFT) is applied at the transmitter side and the Fast Fourier Transform (FFT) is applied at the receiver side.

\section{b. DWT-OFDM System design}

In order to get more improvement of transmission through wireless channel and to keep up with the rapid in-crease in demand of the wireless technologies such as Wi-Fi and the mobile communication, Discrete Wavelet Transform (DWT) have been considered as multicarrier modulation scheme. The Wavelet based OFDM as an alternative for conventional Fourier OFDM in order to reduce the level of ISI and inter-channel interference (ICI) powers.

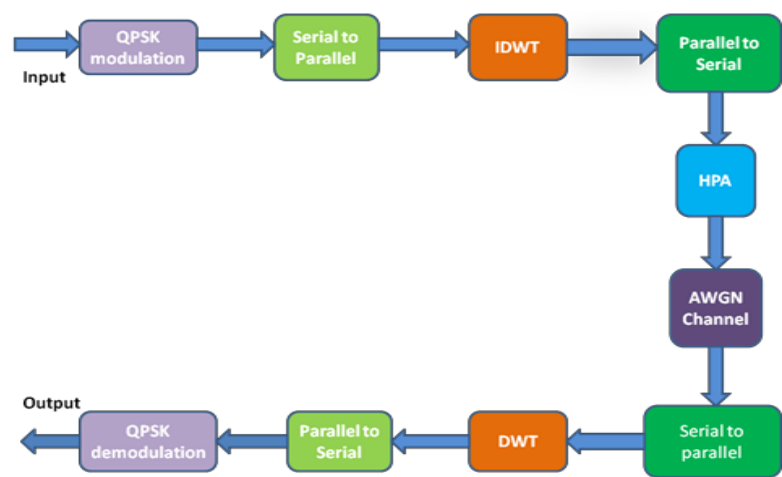

Fig.2.DWT-BasedOFDMSystem

In wireless communication systems design the wavelets have found beneficial applicability, such as channel characterization, interference mitigation, modulation and multiplexing, multiple access communication, Ultra Wide-band (UWB) communication, cognitive radio and networking. In Fig.2 the Discrete Wavelet Transform (DWT) is used in a variety of signal processing applications, such as video compression, Internet communications compression, object recognition and numerical analysis. The power of the wavelet transform comes from the fact that the basic functions of the wavelet are localized in time (or space) and frequency, and have different resolutions in these two domains. IDWT represents the key point of the multicarrier transmitter in which the orthogonal wavelet modulation is performed. Every wavelet basis is seen as a subcarrier and the transmitted symbols are multiplied by these subcarriers.

The orthogonality of the individual basis function prevents inter symbol interference (ISI), furthermore, their mutual orthogonality prevents interference across scales. The transmitted signal $\mathrm{s}(\mathrm{t}) \mathrm{can}$ be expressed as:

$$
S(t)=\sum_{m \in M} \sum_{n} S_{n}^{m} 2^{-m / 2} \psi\left(2^{-m} t-n\right) \quad \rightarrow(3)
$$

Where $\psi_{m, n}(t)$ is the mother wavelet and represent the basis wavelet functions (subcarriers) with compressed factor $\mathrm{m}$ times and shifted $\mathrm{n}$ times for each subcarrier, $S_{n}^{m}$ is the data that is modulated onto the wavelets subcarriers at different scale, and $\mathrm{M}$ represent the number of frequency bands (or number of subcarriers).

At the receiver DWT is performed and the process is inversed. The orthogonality is the key feature that allows sub-carrier separation at the receiver. The orthogonality property leads to make the wavelets orthogonal to each other on both their time position and their frequency localization (scale), such that:

$$
\int \psi_{j, k}(t) \psi_{m, n}(t) d t=\begin{array}{rr}
1 & \text { if } m \& k=n \\
0 & \text { otherwise }
\end{array} \rightarrow(4)
$$

Due to the overlapping nature of wavelets, the wavelet based multicarrier has very high spectral containment and therefore does not need a cyclic prefix to deal with the delay spreads of the channel. Wavelet basis are more spectrally compact than traditional OFDM basis, they have less out of band energy. In other words, the side lobes of the OFDM spectrum, which is a sinc spectrum, contain an important amount of energy that create inter channel interference. By not requiring the cyclic prefix, the available band-width would be more efficiently used, and hence high data rate can be achieved. 


\section{c. DWT-OFDM based STBC system design}

We have introduced a MIMO transmission scheme, called DWT-OFDM-STBC for diversity network, as an alternative to existing techniques such as STBC and VBLAST. The proposed new transmission scheme employs both multi carrier modulation techniques and antenna indices to convey information and exploits the transmit diversity potential of MIMO channels. This paper presents the Discrete Wavelet Transform (DWT) based multicarrier modulation as alternative platform of conventional OFDM in which there is no need for cyclic prefix overhead due to the overlapping nature of DWT. In the STBC scheme, both STBC symbols and the indices of the transmit antennas from which these symbols are transmitted, carry information. We choose Alamouti's STBC, which transmits one symbol per channel use (pcu), as the core STBC due to its advantages in terms of spectral efficiency and simplified ML detection. In Alamouti's STBC, two complex information symbols ( $x 1$ and $x 2)$ drawn from aM-PSK or $M$-QAM constellation are transmitted from two transmit antennas in two symbol intervals in an orthogonal manner by the codeword.

\section{d. Bit Error probability}

In this section, we analyze the error performance of the STBC system, in which $2 m b i t s$ are transmitted during two consecutive symbol intervals using one of the $c M 2=22 m$ different STBC transmission matrices, denoted by $\mathbf{X} 1, \mathbf{X} 2, \ldots, \mathbf{X} 22 m$ here for convenience. An upper bound on the average bit error probability (BEP) is given by the well-known union bound:

$$
P_{b} \leq \frac{1}{2^{2 m}} \sum_{i=1}^{2^{2 m}} \sum_{j=1}^{2^{2 m}} \frac{P\left(X_{i}-X_{j}\right) n_{i, j}}{2 m} \quad \rightarrow(5)
$$

\section{Simulation Results}

The implemented program simulates a $\mathrm{K}$ subcarrier $(\mathrm{K}=16,32,64)$ OFDM system. Constellation mapping or message modulation is performed using MPSK and MQAM. The Additive White Gaussian Noise (AWGN) and Rayleigh channel model is used for this study.

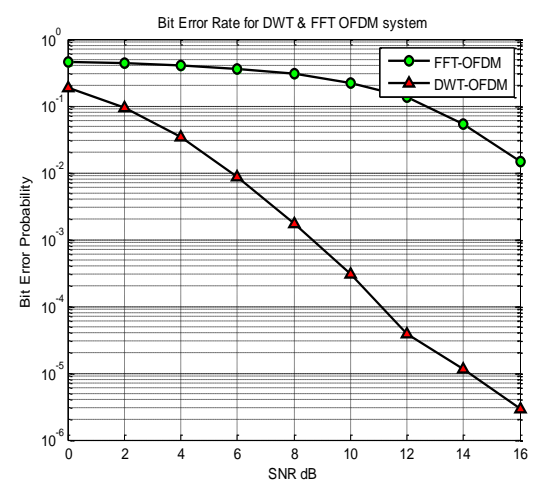

Fig.3.DWT vs. FFT based OFDM system (32 QAM)

In Fig.3 BER vs. SNR plotted. The simulation result shows that the BER performance of the DWT based OFDM system is better than the FFT based OFDM system for (32 Quadrature Amplitude Modulation).

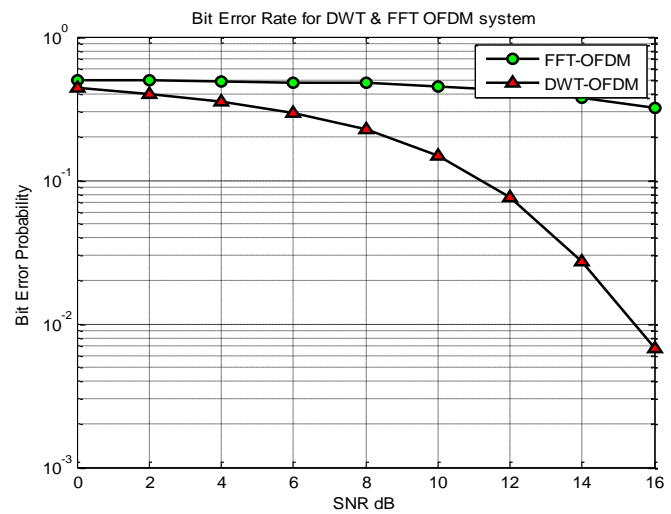

Fig.4.DWT vs. FFT based OFDM system (32 PSK) 
In Fig.4.BER vs. SNR plotted. The simulation result shows that the BER performance of the DWT based OFDM system is better than the FFT based OFDM system for (32 Phase Shift Keying).

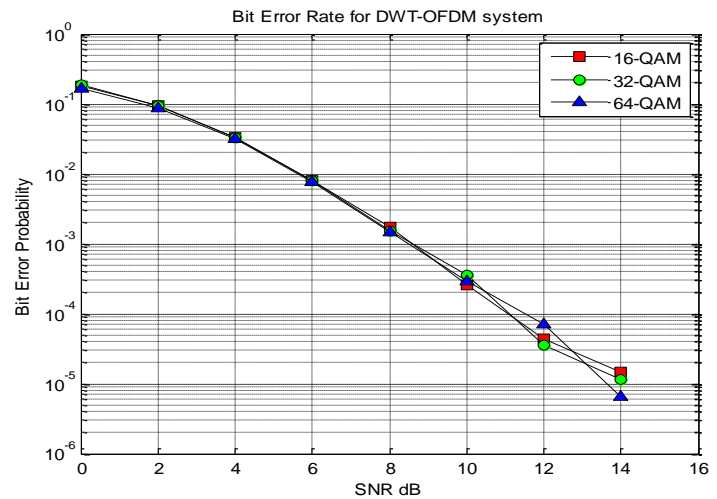

Fig.5.DWT based OFDM system (16, 32, 64 QAM)

In Fig.5 BER vs. SNR plotted. The figure shows the performance comparison between three types of $(16,32,64$ QAM).In that (64-QAM) is gives better performance compare to other two.

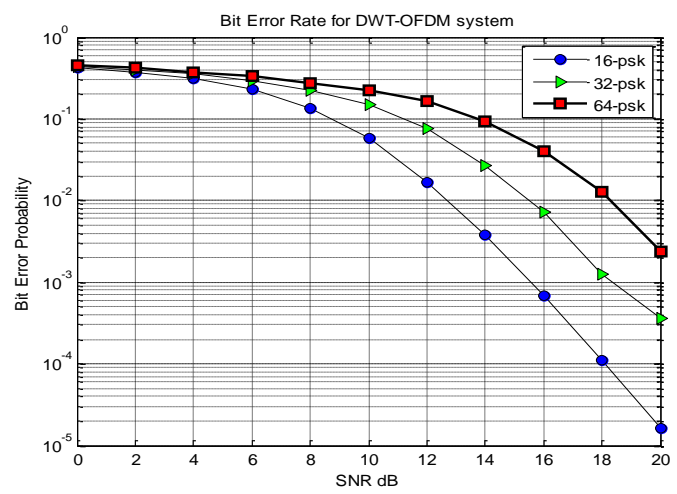

Fig.6.DWT based OFDM system (16, 32, 64 PSK)

In Fig.6 BER vs. SNR plotted. The figure shows the performance comparison between three types of $(16,32,64$ PSK).In that (16-PSK) is gives better performance compare to other two.

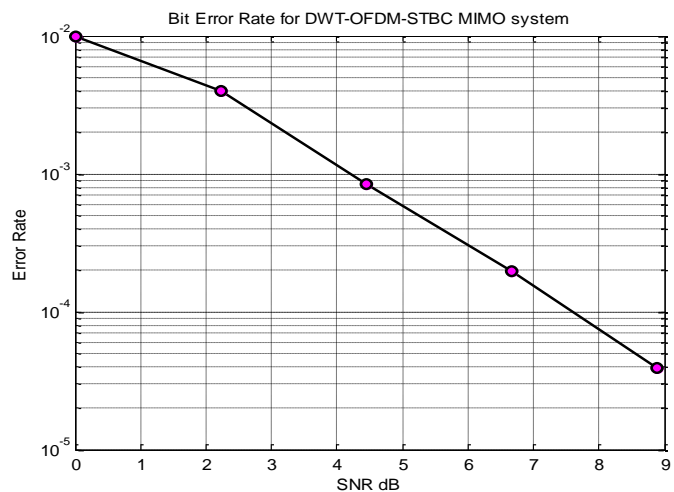

Fig.7.BER for DWT based OFDM-STBC MIMO

InFig.7 shows that advantage of MIMO system in terms of BER, In STBC-MIMO system achieved BER at 0 SNR $\left(10^{-2}\right)$ is better than the BER of conventional OFDM system $\left(10^{-1}\right)$ 


\section{Conclusion}

All the simulation results that we studied whether in SISO or in MIMO-MCM, we found that the performance of the discrete Wavelet transform with 'Haar' mother ('Haar- DWT') based multicarrier modulation are superior to the performance of conventional orthogonal frequency division multiplexing (OFDM) in terms of bit error rate and transmission capacity. Using the DWT as multicarrier modulator in wireless systems rather than using the conventional OFDM (based on FFT) does not need a cyclic prefix extension which represents an overhead, i.e., it reduce the transmission capacity. In other words, in DWT the available bandwidth is wisely utilized and we achieve higher data rate.

MIMO technology improves the data rate in broadband wireless communication. The combination between MIMO and multicarrier modulation schemes improves the BER performance of wireless communications systems. Of course, MIMO with DWT as multicarrier is a very attractive technique for most of modern telecommunication systems that looking for achieve high data rate with small BER. Employing more antennas at the receiver side is the key factor, since it was noticed that the performance of BER increases linearly with the signal to noise ratio, and the system follows Alamouti STBC MIMO scheme due to this performance increases in terms of diversity order and the array gain.

\section{References}

[1]. Swati sharma1 and sanjeev kumar2,"BER performance evaluation of FFT-OFDM and DWT-OFDM", International journal of network and mobile technologies ISSN2229-9114 Electronic version vol2/2011

[2]. W.Saad,N.EI-Fishawy,S.EI-Rabaie,and M.Shokair,"An efficient Technique for OFDM system using DWT" Dept Electronic and communication Engg.,Faculty of Electronics Engg EI-MenufiyaUniversity,EgyptVolume 6104,Dec 2010, pp 533-541.

[3]. Haithamj.taha and M.F.M salleh, "Multicarrier transmission techniques for wireless communication system: A Survey", WSEAS transactions on communication,Issue 5,volume 8,May2009

[4]. $\quad$ E. Basar and Ü. Aygölü, "High-rate full-diversity space-time block codes for three and four transmit antennas," IET Commun., vol. 3, no. 8, pp. 1371-1378, Aug. 2009.

[5]. E. Biglieri, Y. Hong, and E. Viterbo, "On fast-decodable space-time block codes," IEEE Trans. Inf. Theory, vol. 55, no. 2, pp. 524530, Feb. 2009.

[6]. $\quad$ E. Ba sar and Ü. Aygölü, "Full-rate full-diversity STBCs for three and four transmit antennas," Electron. Lett., vol. 44, no. 18, pp. 1076-1077, Aug. 2008

[7]. R. Mesleh, H. Haas, S. Sinanovic, C. W. Ahn, and S. Yun, "Spatial modulation," IEEE Trans. Veh. Technol., vol. 57, no. 4, pp. 2228-2241, July 2008.

[8]. [8] R. Mesleh, H. Haas, C. W. Ahn, and S. Yun, "Spatial modulation-a new low complexity spectral efficiency enhancing technique," in Proc. Conf. Commun.Netw. China, Beijing, China, pp. 1-5, Oct. 2006.

[9]. X.-B. Liang, "Orthogonal designs with maximal rates," IEEE Trans. Inf. Theory, vol. 49, no. 10, pp. 2468-2503, Oct. 2003.

[10]. [10]MukeshTiwari "Effect of channelFading (Rayleigh) in OFDM-STBC Technique"Volume 2, Issue 8,pp.356- 361,August 2012

[11]. SaumyaTripathi, AbhinavRastogi, KapilSachdeva, Mohit Sharma, Pankaj Sharma "PAPR Reduction in OFDM System using DWT with Non-linear High Power Amplifier" ISSN: 2278-3075, Volume-2, Issue-5, April 2013

[12]. GunjanManik, AlkaKalra, SanjeevKalra"Performance Analysis of STBC- OFDM SystemUnder Multipath Fading Channel" ISSN: 2231-2307, Volume-1, Issue-6, January 2012 